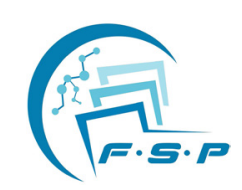

\title{
Comparison of Clinical Characteristics of First and Second Generation Cases of COVID-19
}

\author{
Chao Rui ${ }^{1}$, Liyun Chen ${ }^{1 *}$, Nengjin Gan ${ }^{2}$, Huiming Chen ${ }^{1}$, Fen Huang ${ }^{1}$, Hui He ${ }^{1}$, Zhi Zhang ${ }^{1}$ \\ ${ }^{1}$ Department of Infectious Diseases, The Third People's Hospital of Wuhu City, Wuhu 241000, Anhui, China \\ ${ }^{2}$ Tuberculosis District, The Fourth People's Hospital of Ma'anshan City, Ma'anshan 243000, Anhui, China \\ Email: 119166729@qq.com
}

\begin{abstract}
Purpose - To explore the differences in clinical characteristics of the first and second generation cases of COVID-19. Methods - 63 patients with COVID-19 and asymptomatic infections admitted to the Third People's Hospital of Wuhu City and the Fourth People's Hospital of Ma'anshan City from January 24, 2020 to March 3, 2020 were selected as the research objects. The patients were divided into the first and second generation case groups according to the history of epidemiology, and the differences of clinical data between the two groups were compared. Results - There was no significant difference between the two groups of patients in gender, age, and number of smokers $(P>0.05)$. In the first generation group, the number of people with underlying diseases was lower than that in the second generation group $(86.80 \%$, $92.00 \%)$, and the number of patients of severe or critical type was higher than that in the second generation group (18.40\%, $0.00 \%)$. The difference was statistically significant $(P<0.05)$. The symptoms of the two groups were compared: fever $(84.20 \%, 64.00 \%)$, cough $(78.9 \%, 40.00 \%)$, chest tightness $(44.70 \%, 12.00 \%)$, nausea $(2.60 \%, 36.00 \%)$. The difference was statistically significant $(P<0.05)$. The distribution of lesions in the two groups was compared: bilateral lung lesions $(77.80 \%, 66.70 \%)$, unilateral lesions $(17.80 \%, 5.50 \%)$, and no lesions $(4.40 \%, 27.80 \%)$. The difference was statistically significant $(P<0.05)$. There was no statistically significant difference between the two groups in laboratory tests $(P>0.05)$. Conclusions - Compared with the first generation cases, the second generation cases have different degrees of relief in clinical classification, lower respiratory symptoms and the number of lung lesions; the differentiation of the transmission generation has a certain reference value for the evaluation of the disease condition.
\end{abstract}

Keywords: COVID-19, first generation cases, second generation cases, clinical characteristics

In late December 2019, the COVID-19 epidemic broke out in Wuhan and then spread to all parts of the country. The scope and damage of the epidemic far exceeded the SARS. ${ }^{[1]}$ As of July 27, 2020, there were a total of 83,959 confirmed cases and a total of 4,634 deaths nationwide ${ }^{[2]}$, and there were a total of 990 confirmed cases and a total of 6 deaths in Anhui Province. ${ }^{[3]}$ From an epidemiological point of view, Wuhu City and Ma'anshan City in Anhui Province are dominated by first and second generation cases. In actual clinical work, it was found that there were differences in the severity of the first and second generation cases. Therefore, the article retrospectively analyzes the clinical data of the patients with COVID-19 and asymptomatic infections, and explores the differences in clinical characteristics of patients of different transmission generations, to provide a reference for disease evaluation.

\section{Materials and methods}

\subsection{General materials}

63 patients with COVID-19 and asymptomatic infections admitted to the Third People's Hospital of Wuhu City and the Fourth People's Hospital of Ma'anshan City from January 24, 2020 to March 3, 2020 were selected as the research objects. Inclusion criteria: (1) Meet the diagnostic criteria of the "New Coronavirus Pneumonia Diagnosis and Treatment Plan (Trial Version 1 to 6$)^{\prime} .{ }^{[4]}$ (2) Have a clear epidemiological history.

\subsection{Methods}

Based on the epidemiological history, the research objects were divided into a first generation case group and a second generation case group. Collect the clinical data of the two groups of patients for comparison and analysis. Classification criteria are as follows. ${ }^{[5]}$ The first generation case group refers to the cases with the earliest onset time, that is, the first cases of a clustered epidemic. The situation of asymptomatic infection or latent infection should be comprehensively analyzed and judged by the history of epidemiology and the results of auxiliary examination. The second generation case group must meet the following three conditions at the same time. (1) Only having contact history with first generation cases 
within 14 days after the onset of the disease; (2) No history of living in the epidemic area; (3) No other suspicious exposure history, or no obvious community transmission in the area.

\subsection{Observation indicators}

The patient's general information, such as gender, age, smoking history, and chronic disease history; clinical manifestations, such as respiratory system, digestive system, nervous system symptoms, etc.; laboratory tests, such as blood routine, liver and kidney function, inflammation indicators, myocardial enzymes, etc.; imaging examinations, such as chest CT. All data are entered in parallel and checked to ensure that the original data is true, reliable and traceable.

\subsection{Statistical methods}

The data were statistically analyzed by SPSS 19.0 software; the measurement data of normal distribution were expressed by mean \pm standard deviation; $t$ test was used for comparison between groups; data of skewed distribution were expressed with $\left(P_{25}, P_{75}\right)$; Mann-Whitney test was used for comparison between groups. The counting data were expressed by the number of cases $(\%)$, and the chi-square test was used for comparison between groups. The difference was statistically significant $(P<0.05)$.

\section{Results}

\subsection{Comparison of the general conditions of the two groups of patients}

Among the 63 patients, 38 were in the first generation case group and 25 were in the second generation case group. The age distribution is between 4 and 81 years old; the average age is $43.33 \pm 16.17$ years old; the male to female ratio is $1.33: 1$. There was no significant difference in the distribution of gender, age, and number of smokers between the two groups $(P>0.05)$. The proportion of patients with underlying diseases in the first generation case group was lower than that in the second generation case group, and the difference was statistically significant $(P=0.031)$. See Table 1 .

Table 1. General situation of the two groups of patients [case (\%)]

\begin{tabular}{|c|c|c|c|c|c|}
\hline Item & & $\begin{array}{c}\text { First generation case group } \\
(n=38)\end{array}$ & $\begin{array}{c}\text { Second generation case group } \\
(n=25)\end{array}$ & $\chi^{2}$ & $P$ \\
\hline \multirow{2}{*}{ Gender } & Male & $21(55.30)$ & $15(60.00)$ & \multirow{2}{*}{0.138} & \multirow{2}{*}{0.710} \\
\hline & Female & $17(44.70)$ & $10(40.00)$ & & \\
\hline \multirow{3}{*}{ Age (years old) } & $4 \sim 44$ & $22(57.90)$ & $10(40.00)$ & \multirow{3}{*}{3.938} & \multirow{3}{*}{0.140} \\
\hline & $45 \sim 59$ & $14(36.80)$ & $10(40.00)$ & & \\
\hline & $60 \sim 81$ & $2(5.30)$ & $5(20.00)$ & & \\
\hline \multirow{2}{*}{ Smoking history } & Yes & $5(13.20)$ & $2(8.00)$ & \multirow{2}{*}{$0.052^{*}$} & \multirow{2}{*}{0.820} \\
\hline & None & $33(86.80)$ & $23(92.00)$ & & \\
\hline \multirow{2}{*}{ Underlying disease } & Yes & $6(15.80)$ & $10(40.00)$ & \multirow{2}{*}{4.665} & \multirow{2}{*}{0.031} \\
\hline & None & $32(84.20)$ & $15(60.00)$ & & \\
\hline
\end{tabular}

Note: ${ }^{*}$ Continuity corrected chi-square test

\subsection{Comparison of clinical manifestations of the two groups of patients}

The proportion of severe or critical cases in the first generation case group was higher than that in the second generation case group $(18.40 \%, 0.00 \%)$, and the difference in clinical classification was statistically significant, $P=0.036$. In the first generation case group, the proportion of people with fever $(84.20 \%, 64.00 \%)$, cough $(78.9 \%, 40.00 \%)$, and chest tightness $(44.70 \%, 12.00 \%)$ is higher than that in the second generation case group; the proportion of people with nausea $(2.60 \%, 36.00 \%)$ is lower than that in the second generation case group; the difference is statistically significant $(P<0.05)$. See Table 2 . 
Table 2. Clinical manifestations of the two groups of patients [case (\%)]

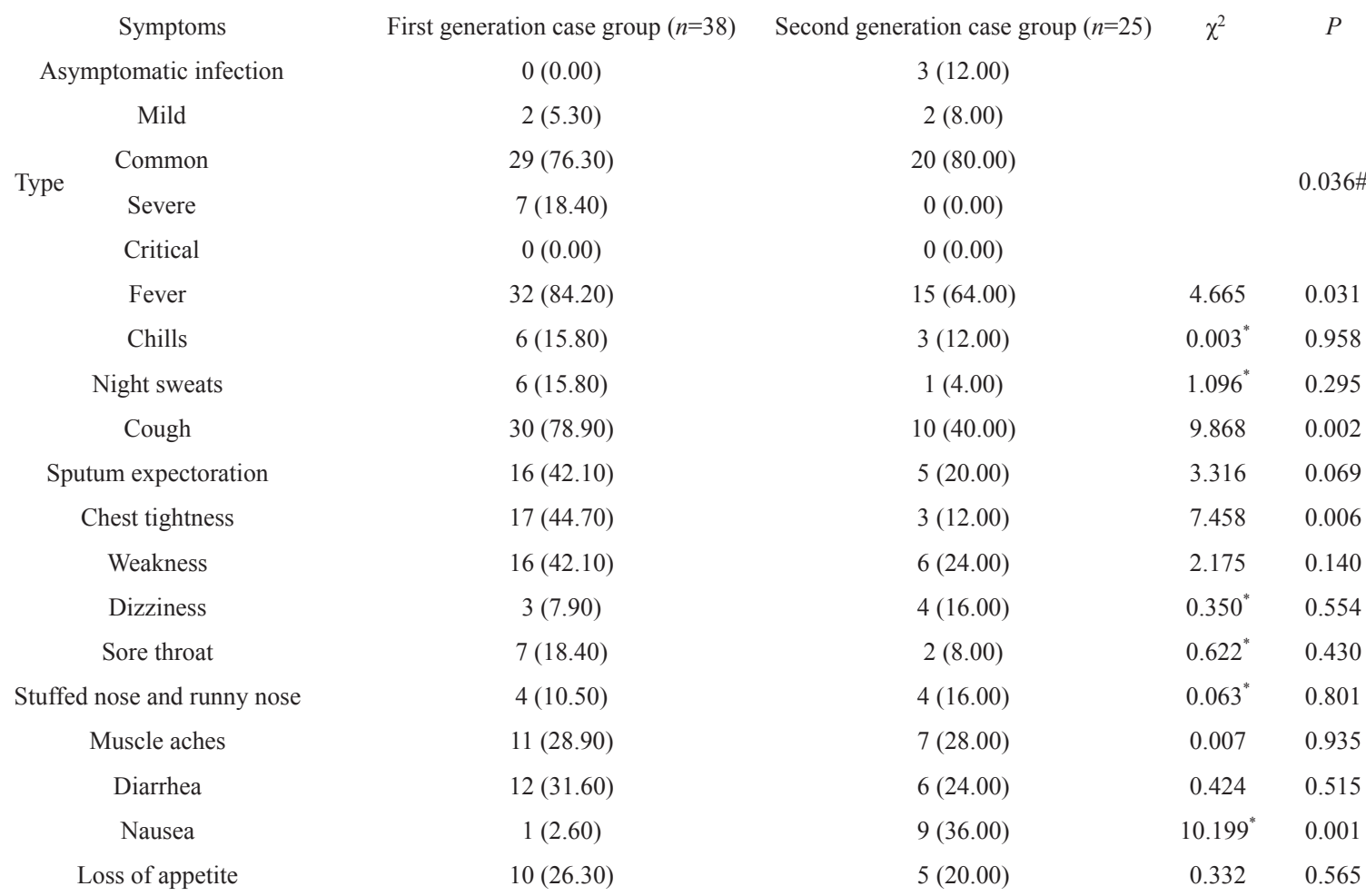

Note: "Continuity corrected chi-square test; \#Fisher exact probability method calculation.

\subsection{Comparison of laboratory tests between the two groups}

Both groups of patients showed normal or decreased white blood cell counts, and decreased lymphocyte counts. Some patients had increased erythrocyte sedimentation rate, C-reactive protein, and lactate dehydrogenase. The difference was not statistically significant $(P>0.05)$. See Table 3 .

Table 3. Laboratory examinations of the two groups of patients (mean \pm standard deviation)

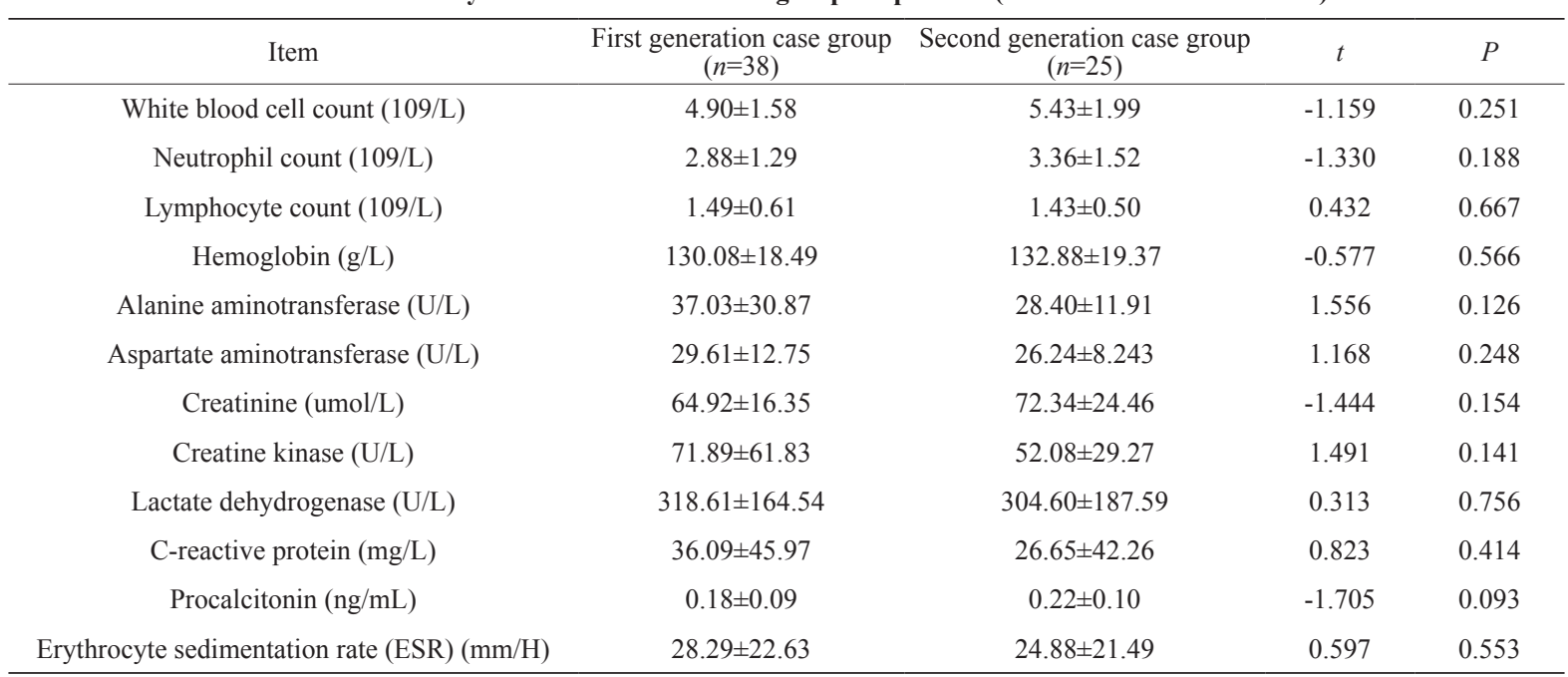

\subsection{Comparison of imaging between the two groups}

There was a statistically significant difference between bilateral lung lesions $(77.80 \%, 66.70 \%)$, unilateral lesions $(17.80 \%, 5.50 \%)$, and no lesions $(4.40 \%, 27.80 \%)(P=0.026)$. In terms of the nature of the lesions, the two groups of lesions were mainly ground-glass shadows, some with consolidation, uneven density, irregular shapes, showing multiple flakes, single flakes and scattered nodules, mainly distributed outside. See Picture 1. 


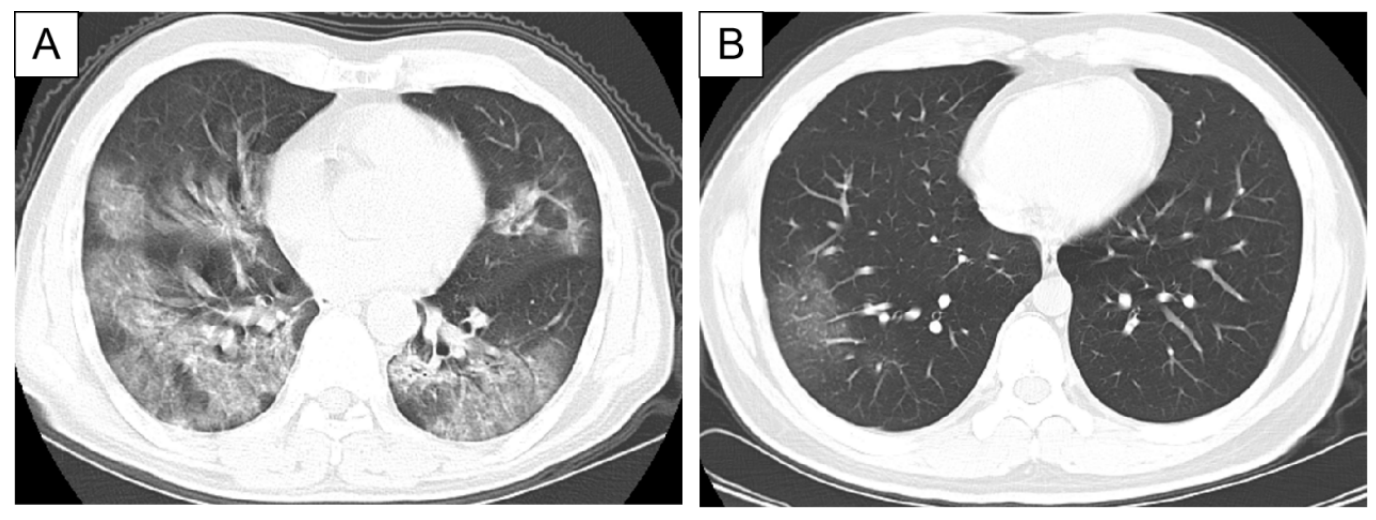

Figure 1. Chest CT findings of two patients

A: A patient of the first generation case. On the second day of admission, the chest CT showed the left lingual lobe, lower lobe, right middle and lower lobes, multiple large pieces of ground glass shadows, and uneven internal density, showing signs of bronchial air and all levels of airway unobstructed. B: A patient of the second generation case. On the second day of admission, the chest CT showed that the lower lobe of the right lung was external, with a large piece of ground glass shadow, uneven internal density, and clear remaining texture.

\section{Discussion}

COVID-19 is an acute respiratory infection caused by a new type of coronavirus infection. The World Health Organization named the virus COVID-19 ${ }^{[6]}$. From a global perspective, COVID-19 is in the epidemic and outbreak phase. It is a new infectious disease, and people's understanding of it is still very limited. Some data show that COVID-19 is an RNA virus with a high mutation rate ${ }^{[7]}$. Disease-causing gene mutation is an important biological characteristic of the continuous spread of viruses, which is manifested as an increase or decrease in virulence. During the struggle between humans and diseases, the level of body-specific immunity will gradually increase. Therefore, as the transmission generation increases, the clinical manifestations may change, but there is still a lack of effective data support. This article retrospectively analyzes the clinical data of the first and second generation cases of COVID-19, in order to have a clearer understanding of the clinical characteristics of the virus after passage.

Studies have shown that the two groups of cases are dominated by common types, but there are 7 severe or critical cases in the first generation group, which is higher than 0 cases in the second generation group. The condition of severe or critical cases progresses rapidly, often accompanied by dyspnea and hypoxemia. In severe cases, life-threatening complications such as acute respiratory distress syndrome, DIC, and shock may occur. Reducing the conversion of common type to severe or critical type is the key to disease treatment. In terms of clinical symptoms, the main manifestations of the two groups are fever $(84.20 \%, 64.00 \%)$ and cough $(78.9 \%, 40.00 \%)$, which are consistent with existing domestic studies ${ }^{[8-11]}$. However, the proportion of people with clinical symptoms in the first generation group was significantly higher than that in the second generation group, and the number of people with chest tightness and the rate of hormone use were also higher than the latter, which indicated that the lower respiratory tract symptoms in the second generation group were less than those in the first generation group. At the same time, both groups had different degrees of "flu-like" symptoms. Muscle aches are more common, but sore throat, nasal congestion, and runny nose are rare, which are in line with the general clinical manifestations of viral infection. However, there was little difference in upper respiratory symptoms between the two groups. During the entire course of the disease, about one-third of the two groups had gastrointestinal reactions, which were mainly manifested as nausea and diarrhea. They were mostly related to adverse drug reactions, and gastrointestinal symptoms were rarely reported. In the second generation cases, the number of patients with nausea was more than that in the first generation cases. This was related to the older age, underlying diseases, and poor drug tolerance of this group of patients, and it was not related to the virus itself.

In laboratory tests, both groups showed decreased or normal white blood cells; decreased absolute lymphocyte values; increased C-reactive protein, erythrocyte sedimentation rate, and lactate dehydrogenase; basically normal procalcitonin. According to Gong Xue's report ${ }^{[8]}$, the decrease in lymphocytes and the increase in C-reactive protein are positively correlated with the severity of the disease. However, in this study, the above-mentioned indicators of the two groups have little difference, and potential differences may be found if the sample size is increased. Chest CT has different manifestations at different stages of the disease. Mainly outside the lesion, it can invade multiple segments. The early 
manifestations are scattered or patchy ground glass shadows, and some progress with consolidation shadows. A small number of fiber streaks appear in the later stage of the disease, but the ground-glass shadows usually accompany the entire disease. This is similar to the research of Chen Zhiyong ${ }^{[12]}$. It was found in the CT that the number of lesions in the firstgeneration group was generally larger, and the area of a single lesion was larger. This is one of the reasons why the lower respiratory tract symptoms of the first-generation cases were heavier than the second-generation cases.

In summary, compared with the first generation cases, the second generation cases have different degrees of reduction in clinical classification, lower respiratory symptoms, and the number of lung lesions. This is partly similar to Wu Wei

[13], s research on the clinical manifestations of SARS in different transmission generations. Therefore, the distinction of transmission generation has certain reference value for the evaluation of the disease.

\section{References}

[1] Riou J, Althaus C L. Pattern of early human-to-human transmission of Wuhan 2019 novel coronavirus (2019-nCoV), December 2019 to January2020. Euro Surveill. 2020; 25(4): 2000058.

[2] National Health Commission of the People's Republic of China. The latest situation of the novel coronavirus pneumonia epidemic situation as of 24:00 on July 27. Available from: http://www. nhc.gov.cn/xcs/yqfkdt/202007/b9807dbfe58c4c718f072594df0f7998.shtml [Accessed 2020-07-28].

[3] Anhui Provincial Health Commission. Anhui Province reported the new crown pneumonia epidemic situation on July 28. Available from: http:/wjw.ah.gov.cn/ztzl/xxgzbdfyyqfk/xxfb/53910451.html [Accessed 2020-07-28].

[4] National Health Commission. New Coronavirus Pneumonia Diagnosis and Treatment Plan (Trial Version 6). Available from: http://www.nhc.gov. cn/yzygj/s7653p/202002/8334a8326dd94d329df351d7da8aefc2.shtml [Accessed 2020-0223].

[5] Chinese Center for Disease Control and Prevention. Technical Guidelines for Epidemiological Investigation of Cluster Epidemics of Novel Coronavirus Pneumonia (Trial Version 1). Chinese Journal of Epidemiology. 2020; 41(3): 293295.

[6] Lu H, Stratton C W, Tang Y W. Outbreak of pneumonia of unknown etiology in Wuhan ,China:the mystery and the miracle. J Med Virol. 2020; 92(4): 401-402.

[7] Wang Ying, Shen Hong, Gao Wenwen, et al. Research progress of novel coronavirus genomics. China Modern Applied Pharmacy. 2020; 37(05): 525-529.

[8] Gong Xue, Mou Fangzheng, Wei Darong, et al. Clinical characteristics and application of traditional Chinese medicine in 225 cases of novel coronavirus pneumonia. World Chinese Medicine. Available from: http://kns.cnki.net/kcms/ detail/ 11.5529.R.20200309.1431.004.html.

[9] Wang Xiaojun, Gao Jing, Wang Xiaobo, et al. Clinical and epidemiological characteristics of new coronavirus pneumonia cases in Gansu Province. Chinese Journal of Infection Control. Available from: http://kns.cnki.net/kcms/detail /43.1390.R.20200316.1437.002.html.

[10] Xiang Tianxin, Liu Jiaming, Xu Fei, et al. Analysis of clinical characteristics of 49 patients with novel coronavirus pneumonia in Jiangxi area. Chinese Journal of Respiratory and Critical Care. Available from: http://kns.cnki.net/ kcms/ detail/51.1631.r.20200228.1506.002.html.

[11] Zhou Shengyu, Wang Chunting, Zhang Wei, et al. Clinical characteristics and treatment effects of 537 patients with new coronavirus pneumonia in Shandong Province. Journal of Shandong University (Medical Edition). Available from: http://kns.cnki.net/kcms/detail/37.1390 .r.20200310.1047.002.html.

[12] Chen Zhiyong, Cheng Zhengyin, Zhang Xuhui, et al. Clinical manifestations and CT imaging characteristics of new coronavirus pneumonia. Radiology Practice. 2020; 35(3): 286-290.

[13] Wu Wei, Wang Jingfeng, Jiang Shanping, et al. Comparison of clinical characteristics of patients with severe acute respiratory syndrome in different transmission generations. Chinese Journal of Internal Medicine. 2004; 43(6): 416419. 